\section{RMD Open}

Rheumatic \& Musculoskeletal Diseases

\title{
Early non-response to certolizumab pegol in rheumatoid arthritis predicts failure to achieve low disease activity at 1 year: data from a prospective observational study
}

To cite: Saraux A, Flipo R-M, Fagnani $\mathrm{F}$, et al. Early nonresponse to certolizumab pegol in rheumatoid arthritis predicts failure to achieve low disease activity at 1 year: data from a prospective observational study. RMD Open 2020;6:e000991. doi:10.1136/ rmdopen-2019-000991

- Additional material is published online only. To view please visit the journal online (http://dx.doi.org/10.1136/ rmdopen-2019-000991).

Received 18 April 2019 Revised 17 October 2019 Accepted 13 November 2019

Check for updates

(C) Author(s) (or their employer(s)) 2020. Re-use permitted under CC BY-NC. No commercial re-use. See rights and permissions. Published by BMJ.

For numbered affiliations see end of article.

Correspondence to Dr Alain Saraux; alain.saraux@chu-brest.fr

\section{ABSTRACT}

Objective To evaluate the performance of clinical criteria for predicting late treatment failure in patients with early non-response to certolizumab pegol (CZP).

Methods A protocol-specified analysis of interim data from ECLAIR, a 3-year longitudinal, prospective, observational, multicentre study of patients with active rheumatoid arthritis (RA) initiating CZP treatment in France, was conducted. Clinical measures assessed were Clinical Disease Activity Index (CDAl), Disease Activity Score-28 with erythrocyte sedimentation rate (DAS28(ESR)) and Health Assessment Questionnaire Disability Index (HAQ-DI). Early non-response was measured at 3 months (M3) and failure to achieve low disease activity (LDA) at 12 months (M12).

Results $574 / 792$ enrolled patients were treated at M3. The numbers available for predictability analyses were 532 (CDAl), 434 (DAS28(ESR)) and 496 (HAQ-DI). Of the three indices evaluated, the highest predictor of non-response value was observed for the CDAl $(88.8 \%$ (95\% Cl 81.0 to 94.1$)$ ), indicating that up to $88 \%$ of patients identified as non-responders at M3 failed to achieve LDA at M12, regardless of baseline disease severity or treatment history. The specificity for this measure was also very high $(96.0 \%)$, indicating that less than $5 \%$ of patients who achieved CDAl response at M12 had not responded at M3. Similar predictability was observed for DAS28(ESR), but only in patients with high disease activity at baseline and/or those previously treated by a biological diseasemodifying antirheumatic drug.

Conclusion CDAl non-response at M3 is a predictor of failure to achieve the therapeutic target of LDA at M12 in patients with RA initiating treatment with CZP.

\section{INTRODUCTION}

Rheumatoid arthritis (RA) is a chronic systemic autoimmune disease characterised by synovitis of the peripheral joints, swelling, stiffness, pain, structural damage to joints,

\section{Key messages}

What is already known about this subject?

- Rheumatoid arthritis (RA) treatment guidelines recommend following a 'treat-to-target' strategy and, in cases of inadequate response, switching to another treatment.

- To adapt treatment, early clinical markers of response or non-response are needed. However, there is a lack of such markers as well as data on how they perform in clinical practice.

What does this study add?

- This study revealed that early non-response (at month 3) based on Clinical Disease Activity Index (CDAl) is a good predictor of late treatment failure (at month 12) in patients initiating certolizumab pegol in real-world clinical practice.

- Similar predictability was observed for Disease Activity Score-28 with erythrocyte sedimentation rate (DAS28(ESR)) in patients with high disease activity at baseline and/or those previously treated by a biological disease-modifying antirheumatic drug.

How might this impact on clinical practice?

- CDAl and DAS28(ESR) could be used to ensure that patients are not unnecessarily exposed to ineffective therapy, by identifying likely failure at 3 months.

functional disability and impaired healthrelated quality of life. ${ }^{12}$ Collectively, these result in significant morbidity and mortality compared with the healthy population.

The aim of RA treatment is to decrease inflammation, relieve symptoms of pain and discomfort, slow the progression of joint damage, and maintain the patient's quality of life and ability to function. ${ }^{1-3}$ As specified in the most recent EULAR RA management 
recommendations, the treatment goal is to achieve a target of sustained remission (Disease Activity Score-28 with erythrocyte sedimentation rate (DAS28(ESR)) $<2.6$ ) or low disease activity (DAS28(ESR) <3.2) in every patient. ${ }^{3}$ The introduction of disease-modifying antirheumatic drugs (DMARDs), a class of therapeutic agents that includes both conventional synthetic drugs (csDMARDs) and biological agents (bDMARDs), has allowed these goals to be met partially. ${ }^{4}$ However, the treatment response to DMARDs is heterogeneous in clinical practice. ${ }^{5}$ A meta-analysis of combination and monotherapy treatments in DMARD-experienced patients with RA showed that in randomised clinical trials, less than $50 \%$ of patients receiving therapy met the American College of Rheumatology (ACR) 50 criteria for treatment response. ${ }^{6}$ Response rates to DMARDs are potentially lower in a real-world setting. ${ }^{7}$

For this reason, current RA treatment guidelines recommend following a 'treat-to-target' strategy, whereby patients should be treated with DMARDs as early as possible after diagnosis, monitored at 3 monthly intervals and, in cases of inadequate response, switched to another csDMARD or bDMARD. ${ }^{38}$ Anti-tumour necrosis factors (TNFs) have revolutionised the management of chronic inflammatory diseases including RA, ankylosing spondylitis, psoriatic arthritis, psoriasis and Crohn's disease. ${ }^{9}$ However, despite good responder rates among patients with RA, significant variability is noted in patients' response to treatment. ${ }^{10}$ The 'treat-to-target' strategy for RA requires the use of reliable, early clinical markers of response or non-response in order to adapt treatment. In particular, early markers of treatment failure could be used to ensure that patients are not unnecessarily exposed to ineffective therapy. Several post hoc analyses $^{11} 12$ of data from RAPID-1, a phase III trial of the anti-TNF therapy certolizumab pegol (CZP), ${ }^{13}$ have shown that non-response at 3 months (M3), as determined by DAS28, Clinical Disease Activity Index (CDAI), ACR50 and Health Assessment Questionnaire Disability Index (HAQ-DI), has high specificity for predicting treatment failure at 12 months (M12). While this has been investigated previously by Aletaha et al (with methotrexate, biologicals or combination) ${ }^{14}$ and Keystone et al (with adalimumab), ${ }^{15}$ these studies operated within a clinical trial context, and how these indicators would perform in real-world treatment settings is unknown, as no pertinent data from prospective observational studies are available. The present study may be distinguished by its real-world setting.

Significant improvements in long-term outcome have been demonstrated following the implementation of the 'treat-to-target' strategy, ${ }^{16}$ particularly in the present context of a growing biological therapeutic arsenal with various modes of action.

Our aim was to evaluate the performance of different clinical criteria for early non-response to CZP as predictors of late treatment failure in routine practice.

\section{METHODS}

This was a protocol-specified analysis of interim (12month) data from the ECLAIR (Evaluation de l'évolution des patients traités par certolizumab pegol pour une polyarthrite rhumatoïde en pratique courante en France) study. ECLAIR was a 3-year, longitudinal, prospective, observational, multicentre study of patients with active RA initiating CZP treatment, following a request from the French National Authority for Health (HAS) to describe the effectiveness and tolerability of this agent in current medical practice.

Under conditions of everyday clinical practice, the current study provided an opportunity to evaluate the performance of different clinical criteria for early nonresponse to CZP (at M3) as predictors of late treatment failure (at M12). The clinical measures chosen for both early non-response and late treatment failure were CDAI, ${ }^{17}{ }^{18}$ DAS28 (ESR) ${ }^{19}$ and HAQ-DI. ${ }^{20}$

The study was conducted by hospital rheumatologists and internal medicine specialists who treat and monitor patients with RA in France. Patients were enrolled between December 2011 and December 2013.

\section{Study population}

Participating physicians

Physicians were identified from an exhaustive list of hospital rheumatologists and internal medicine specialists who treat and monitor patients with RA. Physicians were contacted by post and invited to participate in the study; those who did not reply were contacted by telephone. Recruitment ended as soon as all physicians on the list had been contacted. Around 250 physicians were anticipated to participate in the study.

\section{Patient population}

Each participating physician was expected to include the first three consecutive patients who were seen in routine consultation, fulfilled the eligibility criteria and agreed to participate in the study. The study population comprised adult patients with moderate-to-severe active RA, for whom the physician decided to initiate first treatment with CZP according to the prescribing information defined in the Summary of Product Characteristics. Patients previously treated with CZP, those recently (within the previous 3 months) or currently participating in a clinical study, and those who were not expected to be able to adhere to the study plan (- to understand and complete the questionnaires, to respect the study visit schedule or to be compliant with the medication) were excluded from the study.

\section{Study procedures}

As this was an observational study, no specific study procedures were required in the protocol. Treatment with CZP was expected to follow the prescribing recommendations, namely a loading dose of $400 \mathrm{mg}$ at weeks 0 , 2 and 4 , followed by a maintenance dose of $200 \mathrm{mg}$ every 2 weeks thereafter. 
Table 1 Definitions of non-response and of predictability indices used in the study

\begin{tabular}{|c|c|c|c|}
\hline \multicolumn{4}{|l|}{ Definitions of non-response ${ }^{9}$} \\
\hline Outcome at $\mathrm{M}^{*}$ & CDAI & $\triangle \mathrm{DAS} 28(\mathrm{ESR})$ & $\triangle \mathrm{HAQ}-\mathrm{DI}$ \\
\hline Early non-response & Score $>22$ & $\begin{array}{l}\text { Decrease of } \leq 1.2 \text { compared with baseline } \\
\text { value }\end{array}$ & $\begin{array}{l}\text { Decrease of }<0.22 \text { compared with baseline } \\
\text { value }\end{array}$ \\
\hline Outcome at $\mathrm{M} 12^{*}$ & CDAI & DAS28(ESR) & HAQ-DI \\
\hline Treatment failure & Score $>10$ & Score $>3.2$ & Score $>0.5$ \\
\hline \multicolumn{4}{|c|}{ Definitions of indices of predictability } \\
\hline Sensitivity & \multicolumn{3}{|c|}{ In all patients in treatment failure at $\mathrm{M} 12$, the proportion with early non-response observed at $\mathrm{M} 3$} \\
\hline Specificity & \multicolumn{3}{|c|}{ In all patients with treatment success at M12, the proportion with early response observed at M3 } \\
\hline Predictability of non-response & \multicolumn{3}{|c|}{ In all patients identified as non-responders by M3, the proportion with treatment failure at M12 } \\
\hline Predictability of response & \multicolumn{3}{|c|}{ In all patients identified as responders by $\mathrm{M} 3$, the proportion with treatment success at M12 } \\
\hline Accuracy & \multicolumn{3}{|c|}{$\begin{array}{l}\text { In all patients, the proportion for whom treatment outcome (response or non-response) at M3 is the } \\
\text { same as at M12 (either failure or success) }\end{array}$} \\
\hline
\end{tabular}

${ }^{*}$ The CDAl criterion assessed at M3 and M12 was the absolute value of the score. For the DAS28(ESR) and the HAQ-DI, the criterion was the change in score since baseline at $\mathrm{M} 3$ and the absolute value of the score at M12.

CDAI, Clinical Disease Activity Index; CZP, certolizumab pegol; DAS28, 28-joint Disease Activity Score; ESR, erythrocyte sedimentation rate; HAQ-DI, Health Assessment Questionnaire Disability Index; M3, 3 months; M12, 12 months.

Data were collected during the normal course of patient care and in accordance with national recommendations for the management of RA. ${ }^{2}$ Data were collected at the baseline visit and then at around 3,6,12, 18, 24 and 36 months thereafter. Only data from the first 12 months were considered in this interim analysis. The visits at baseline and at M12 were overseen by the hospital rheumatologist or the internal medicine specialist who prescribed CZP, whereas the visits at M3 and M6 could have been overseen by hospital or community rheumatologists, internal medicine specialists or general practitioners.

Disease activity was assessed at each visit using CDAI, DAS28(ESR) and HAQ-DI.

\section{Definitions of non-response}

Outcomes at 3 and 12 months (M3 and M12) after initiation of first treatment with CZP were assessed. The predictability indices and definitions of non-response for CDAI, DAS28(ESR) and HAQ-DI used in this study are listed in table 1; these definitions are identical to those used in a similar post hoc analysis of the RAPID-1 study. ${ }^{11}$ While remission is the preferred treatment target in clinical practice, achievement of low disease activity (LDA) serves as an alternative for those with established disease. ${ }^{8}$ Thus, failure to achieve LDA was used to define treatment failure at M12 in the present study.

\section{Statistical analysis}

The analysis population was defined as all enrolled patients who had received at least one dose of CZP and who met the eligibility criteria for the study. Pre-specified subgroup analyses were also performed in patients with high disease activity (DAS28 (ESR) score $>5.1$ at baseline) and in patients with no bDMARD treatment prior to CZP initiation, to facilitate comparison with the post hoc analysis of the RAPID-1 study. ${ }^{11}$
Patients with no data available at M3 (missing visit or missing data), who were no longer taking CZP at M3 or who had temporarily discontinued for more than two continuous weeks before the visit at M3 were excluded from the analyses. DAS28(ESR) and CDAI missing data at M12 were imputed by linear interpolation between the score of the previous and the next visit if both were available, or last observation carried forward. Failure was imputed at M12 in cases of permanent CZP discontinuation between M3 and M12.

Predictive performance was assessed by evaluating the relationship between early non-response at M3 and treatment failure at M12. For each clinical measure, the sensitivity, specificity, and predictability of response and non-response were determined with $95 \%$ CIs. The predictability of non-response (the proportion of treatment failures who were identified as non-responders at M3) was considered the principal analysis objective.

Statistical analysis and generation of tables and patient data listings were performed using SAS V.9.2.

\section{Ethical considerations}

The study was conducted in conformity with the Guidelines for Good Epidemiology Practice and the ethical principles specified in the Declaration of Helsinki (revised in 1989) including all subsequent amendments and all relevant national legislation. As this study was observational and did not modify the care received by the patient, ethical committee approval was not required. All patient data were anonymised. All patients were informed of the goals of the study prior to inclusion. Approval was obtained from the Advisory Committee on Information Processing for Health Research with respect to confidentiality (CCTIRS-no. 11.446) and from the French National Commission for Data Processing and Privacy 


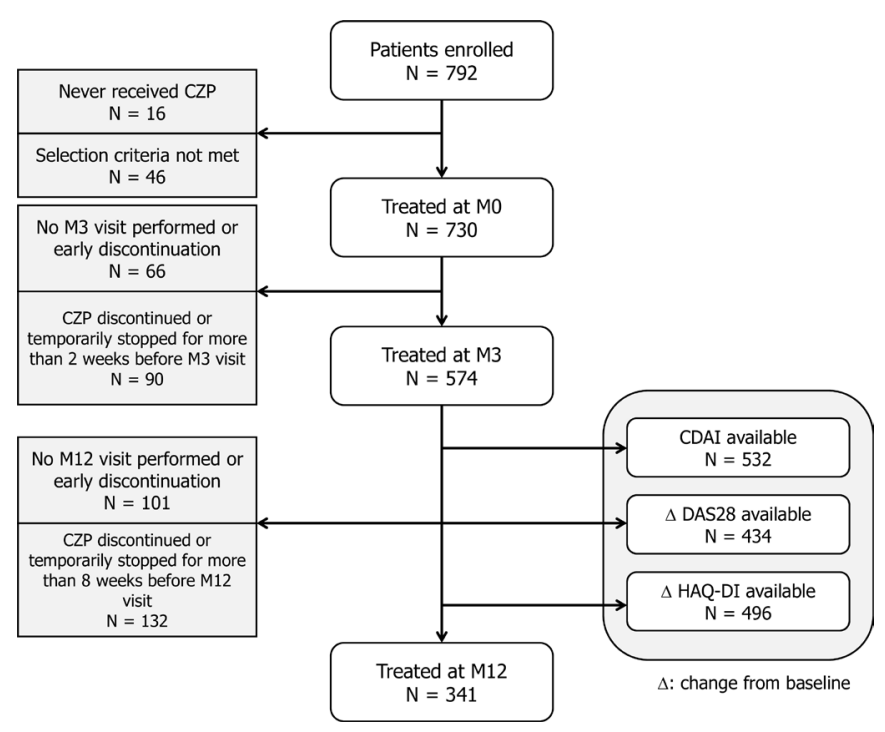

Figure 1 Patient flow diagram. CDAl, Clinical Disease Activity Index; CZP, certolizumab pegol; DAS28(ESR), Disease Activity Score-28 (erythrocyte sedimentation rate); HAQ-DI, Health Assessment Questionnaire Disability Index; M0, baseline; M3, 3 months; M12, 12 months.

(Commission Nationale de l'Informatique et des Libertés (CNIL-no. 911360)).

\section{RESULTS}

\section{Participating physicians}

Physicians were selected from an exhaustive list of hospital rheumatologists and internal medicine specialists who treat and follow up patients with RA in France, provided by CEGEDIM (Centre de gestion, de documentation, d'informatique et de marketing).

In total, 327 rheumatologists and 29 internal medicine specialists were included in the study (in addition to 9 whose specialty was not reported); 220 had an exclusively hospital-based practice.

Of the 365 physicians, 176 enrolled patients and returned at least one case report form. As the number of participating physicians was lower than expected, the protocol was amended to authorise inclusion of up to 20 patients per physician (instead of the three initially specified). The mean number of patients included per physician was 4.5 (range, 1-20).

\section{Patients}

Overall, 792 patients were enrolled in the study, of whom $730(92.2 \%)$ were analysed (figure 1). Follow-up data at M3 were available for 574 patients. The remaining 156 were excluded from the analysis as no M3 visit was performed (66 patients), CZP was discontinued (59 patients) or CZP was temporarily stopped for more than 2 weeks before the M3 visit (31 patients). The number of patients available for predictability analyses were 532 for CDAI, 434 for DAS28(ESR) and 496 for HAQ-DI scores at M3. Follow-up data after treatment at M12 were available for 341 patients for all measures.
Baseline characteristics of the 730 patients eligible for analysis are presented in table 2. Patients were aged $55.0 \pm 13.1$ years and mainly female $(77.9 \%)$. Of the 643 patients with a DAS28 score documented at baseline, 263 $(40.9 \%)$ had high disease activity. No prior bDMARD exposure was reported for 490 patients $(67.1 \%)$.

\section{Early non-response and late treatment failure rates}

Rates of non-response at M3 and treatment failure at M12, together with the performance indices for each clinical measure, are presented in table 3.

At M3, the proportion of patients classified as nonresponders varied depending on the index considered, ranging from $16.7 \%$ for the CDAI to $46.8 \%$ for the HAQ-DI score. At M12, the proportions of patients in treatment failure (not achieving LDA) were $53.4 \%$ and $54.8 \%$ for CDAI and DAS28(ESR), respectively, and $66.3 \%$ for HAQ-DI.

\section{Predictability of treatment failure at M12}

Of the three indices evaluated, the highest predictability of non-response (PoNR) value was observed for the CDAI measure. The PoNR for this measure was $88.8 \%$ (95\% CI 81.0 to 94.1 ), indicating that the majority of patients identified as non-responders at M3 failed to respond at M12. The specificity was also very high $(96.0 \%)$, indicating that less than $5 \%$ of patients who achieved CDAI response at M12 had not responded at M3 (table 3).

Interestingly, the performance of the CDAI measure was similar between patients with high disease activity at baseline and those with low/moderate disease activity (figure 2A), and between those who were bDMARD-naive (figure 2B, online supplementary table 4) and those who had already been treated with a bDMARD (online supplementary table 5). In contrast, for the DAS28(ESR) and HAQ-DI measures, the PoNR was higher in patients with high disease activity and prior bDMARD treatment (online supplementary table 3), as well as the overall subgroup of patients with high disease activity at baseline (online supplementary table 1 ), and to a lesser extent, the overall subgroup of patients with prior bDMARD treatment (online supplementary table 5). This means that both disease severity at baseline and treatment history had an impact on the performance of these two measures as predictors of long-term treatment failure.

\section{DISCUSSION}

This study was the first conducted in patients with RA treated with CZP in a real-world setting in France, and demonstrated a relationship between early non-response to treatment and late treatment failure, based on CDAI.

Although anti-TNF therapy can have a positive impact on outcomes in patients with RA, some patients do not experience significant improvements in disease activity. In the absence of baseline factors to predict future treatment response, ${ }^{14}$ models have focused on using data collected at an early timepoint in the course of treatment (within 3 months or sooner after initiating an anti-TNF) 
Table 2 Patient characteristics at baseline

\begin{tabular}{|c|c|c|c|}
\hline & Patients treated at M0 & Patients treated at M3 & $\begin{array}{l}\text { Patients not treated at } \\
\text { M3 }\end{array}$ \\
\hline & $n=730$ & $n=574$ & $n=156$ \\
\hline \multicolumn{4}{|l|}{ Age at baseline (years) } \\
\hline Mean \pm SD & $55.0 \pm 13.1$ & $55.2 \pm 13.1$ & $54.4 \pm 13.0$ \\
\hline Gender (women; n (\%)) & $569(77.9 \%)$ & $442(77.0 \%)$ & $127(81.4 \%)$ \\
\hline \multicolumn{4}{|l|}{ Body mass index (kg/m²) } \\
\hline Women; mean $\pm S D$ & $25.25 \pm 5.37(n=558)$ & $25.23 \pm 5.40(n=435)$ & $25.31 \pm 5.29(n=123)$ \\
\hline Men; mean $\pm S D$ & $25.52 \pm 3.99(n=156)$ & $25.62 \pm 3.84(n=128)$ & $25.04 \pm 4.68(n=28)$ \\
\hline Disease duration (years) & $(n=728)$ & $(n=572)$ & $(n=156)$ \\
\hline Mean \pm SD & $8.79 \pm 8.79$ & $8.63 \pm 8.86$ & $9.41 \pm 8.50$ \\
\hline Tender/painful joint count (28 joints) & $(n=721)$ & $(n=568)$ & $(n=153)$ \\
\hline Mean \pm SD & $8.93 \pm 7.04$ & $8.71 \pm 6.96$ & $9.74 \pm 7.27$ \\
\hline Swollen joint count (28 joints) & $(n=719)$ & $(n=567)$ & $(n=152)$ \\
\hline Mean $\pm S D$ & $5.64 \pm 5.05$ & $5.60 \pm 4.99$ & $5.77 \pm 5.30$ \\
\hline CDAl score & $(n=698)$ & $(n=549)$ & $(n=149)$ \\
\hline Mean \pm SD & $25.72 \pm 12.35$ & $25.17 \pm 12.29$ & $27.73 \pm 12.39$ \\
\hline DAS28(ESR) score & $(n=643)$ & $(n=509)$ & $(n=134)$ \\
\hline Mean \pm SD & $4.84 \pm 1.27$ & $4.79 \pm 1.27$ & $5.05 \pm 1.27$ \\
\hline 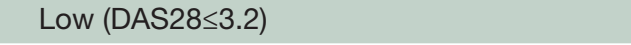 & $62(9.6 \%)$ & $52(10.2 \%)$ & $10(7.5)$ \\
\hline Moderate $(3.2 \leq \mathrm{DAS} 28 \leq 5.1)$ & $318(49.5 \%)$ & $256(50.3 \%)$ & $62(46.3)$ \\
\hline High (DAS28>5.1) & $263(40.9 \%)$ & $201(39.5 \%)$ & $62(46.3)$ \\
\hline HAQ-DI total score & $(n=692)$ & $(n=545)$ & $(n=147)$ \\
\hline Mean $\pm S D$ & $1.27 \pm 0.69$ & $1.23 \pm 0.69$ & $1.42 \pm 0.69$ \\
\hline Rheumatoid factor & $(\mathrm{n}=378)$ & $(n=314)$ & $(n=64)$ \\
\hline Positive, n (\%) & $263(69.6 \%)$ & $218(69.4 \%)$ & $45(70.3 \%)$ \\
\hline Anti-CCP antibodies & $(n=396)$ & $(n=324)$ & $(n=72)$ \\
\hline Positive, n (\%) & $292(73.7 \%)$ & $237(73.1 \%)$ & $55(76.4 \%)$ \\
\hline $\begin{array}{l}\text { Antirheumatic drug treatments prior to CZP } \\
\text { initiation }\end{array}$ & $(n=730)$ & $(n=574)$ & $(n=156)$ \\
\hline No DMARDs & $8(1.1 \%)$ & $7(1.2 \%)$ & $1(0.6 \%)$ \\
\hline Conventional synthetic DMARDs & $712(97.5 \%)$ & $560(97.6 \%)$ & $152(97.4 \%)$ \\
\hline Methotrexate & $682(93.4 \%)$ & $540(94.1 \%)$ & $142(91.0 \%)$ \\
\hline Biological DMARDs & 240 (32.9\%) & $175(30.5 \%)$ & $65(41.7 \%)$ \\
\hline Anti-TNF agents & $221(30.3 \%)$ & $163(28.4 \%)$ & $58(37.2 \%)$ \\
\hline NSAIDS & $403(55.2 \%)$ & $314(54.7)$ & $89(57.1)$ \\
\hline Glucocorticoids & $562(77.0 \%)$ & $444(77.4)$ & $118(75.6)$ \\
\hline $\begin{array}{l}\text { Antirheumatic drug treatments concomitant to } \\
\text { CZP initiation }\end{array}$ & $(n=730)$ & $(n=574)$ & $(n=156)$ \\
\hline No DMARDs & $259(35.5 \%)$ & $203(35.4 \%)$ & $56(35.9 \%)$ \\
\hline Conventional synthetic DMARDs & $471(64.5 \%)$ & $371(64.6 \%)$ & $100(64.1 \%)$ \\
\hline Methotrexate & $391(53.6 \%)$ & $315(54.9 \%)$ & $76(48.7 \%)$ \\
\hline Leflunomide & $63(8.6 \%)$ & $43(7.5 \%)$ & $20(12.8 \%)$ \\
\hline Hydroxychloroquine & $21(2.9 \%)$ & $16(2.8 \%)$ & $5(3.2 \%)$ \\
\hline Sulfasalazine & $17(2.3 \%)$ & $14(2.4 \%)$ & $3(1.9 \%)$ \\
\hline NSAIDS & $258(35.3 \%)$ & $198(34.5)$ & $60(38.5)$ \\
\hline Glucocorticoids & $373(51.1 \%)$ & $288(50.2)$ & $85(54.5)$ \\
\hline
\end{tabular}


Table 2 Continued

\begin{tabular}{|c|c|c|}
\hline Patients treated at MO & Patients treated at M3 & $\begin{array}{l}\text { Patients not treated at } \\
\text { M3 }\end{array}$ \\
\hline$n=730$ & $n=574$ & $n=156$ \\
\hline
\end{tabular}

CCP, cyclic citrullinated peptide; CDAI, Clinical Disease Activity Score; DAS28, 28-joint Disease Activity Score; DMARD, diseasemodifying antirheumatic drug; ESR, erythrocyte sedimentation rate; HAQ-DI, Health Assessment Questionnaire Disability Index; NSAID, non-steroidal anti-inflammatory drug; TNF, tumour necrosis factor.

to predict the attainment of long-term therapeutic targets. ${ }^{21} 22$ Previous studies looking at positive predictors of long-term response in patients with RA have already assessed whether an association exists between early and long-term treatment response (remission or LDA) in clinical trials, ${ }^{121-23}$ and in real-world settings. ${ }^{24}$ However, for patients who will not achieve long-term treatment targets, it might be more relevant to predict treatment failure as early as possible, so that therapy can be adjusted to optimise the long-term clinical outcome.
This study aimed to characterise the performance of different measures of early non-response as predictive markers of late treatment failure (defined as failure to achieve LDA) in unselected patients with RA treated with CZP. The PoNR value was considered the principal analysis index; this indicates the probability of a patient with early non-response (at 3 months: M3) failing to achieve later treatment targets (at 12 months: M12).

For the CDAI, we observed high values both for predictability of long-term treatment failure (89\%) and

Table 3 Early (M3) and late (M12) treatment failure rates-overall population

\begin{tabular}{|c|c|c|c|}
\hline \multicolumn{4}{|c|}{ CDAl at M12 } \\
\hline CDAl at M3 & >10 (late treatment failure) & $\begin{array}{l}\leq 10 \text { (late treatment } \\
\text { response) }\end{array}$ & Total \\
\hline >22 (early treatment non-response) & 79 & 10 & 89 \\
\hline$\leq 22$ (no early treatment non-response) & 205 & 238 & 443 \\
\hline \multirow[t]{3}{*}{ Total } & 284 & 248 & 532 \\
\hline & \multicolumn{3}{|c|}{$\begin{array}{l}\text { Sensitivity=27.8\% (79/284), } 95 \% \mathrm{Cl} 22.8 \text { to } 33.2 \\
\text { Specificity=96.0\% (238/248), } 95 \% \mathrm{Cl} 93.0 \text { to } 97.9 \\
\text { PoNR=88.8\% (79/89), } 95 \% \mathrm{Cl} 81.0 \text { to } 94.1 \\
\text { PoR=53.7\% (238/443), 95\% Cl } 49.1 \text { to } 58.3\end{array}$} \\
\hline & \multicolumn{3}{|l|}{ DAS28(ESR) at M12 } \\
\hline$\triangle \mathrm{DAS28}(\mathrm{ESR})$ at M3 & $>3.2$ (late treatment failure) & $\begin{array}{l}\leq 3.2 \text { (late treatment } \\
\text { response) }\end{array}$ & Total \\
\hline$<1.2$ (early treatment non-response) & 136 & 61 & 197 \\
\hline$\geq 1.2$ (no early treatment non-response) & 102 & 135 & 237 \\
\hline \multirow[t]{3}{*}{ Total } & 238 & 196 & 434 \\
\hline & \multicolumn{3}{|c|}{$\begin{array}{l}\text { Sensitivity=57.1\% (136/238), 95\% Cl } 50.8 \text { to } 63.3 \\
\text { Specificity=68.9\% (135/196), 95\% Cl } 62.2 \text { to } 75.0 \\
\text { PoNR=69.0\% (136/197), 95\% Cl } 62.3 \text { to } 75.2 \\
\text { PoR }=57.0 \%(135 / 237), 95 \% \mathrm{Cl} 50.6 \text { to } 63.1\end{array}$} \\
\hline & \multicolumn{3}{|l|}{ HAQ-DI at M12 } \\
\hline$\triangle \mathrm{HAQ}-\mathrm{DI}$ at $\mathrm{M} 3$ & $>0.5$ (late treatment failure) & $\begin{array}{l}\leq 0.5 \text { (late treatment } \\
\text { response) }\end{array}$ & Total \\
\hline$<0.22$ (early treatment non-response) & 175 & 57 & 232 \\
\hline$\geq 0.22$ (no early treatment non-response) & 154 & 110 & 264 \\
\hline \multirow[t]{2}{*}{ Total } & 329 & 167 & 496 \\
\hline & \multicolumn{3}{|c|}{$\begin{array}{l}\text { Sensitivity=53.2\% (175/329), } 95 \% \mathrm{Cl} 47.8 \text { to } 58.5 \\
\text { Specificity=65.9\% (110/167), 95\% Cl } 58.5 \text { to } 72.7 \\
\text { PoNR=75.4\% (175/232), 95\% Cl } 69.6 \text { to } 80.6 \\
\text { PoR=41.7\% (110/264), } 95 \% \mathrm{Cl} 35.8 \text { to } 47.7\end{array}$} \\
\hline
\end{tabular}

CDAI, Clinical Disease Activity Index; CZP, certolizumab pegol; $\triangle \mathrm{DAS28(ESR),} \mathrm{change} \mathrm{in} \mathrm{28-joint} \mathrm{Disease} \mathrm{Activity} \mathrm{Score} \mathrm{(erythrocyte}$ sedimentation rate); $\triangle \mathrm{HAQ}-\mathrm{DI}$, change in Health Assessment Questionnaire Disability Index;M3, 3 months; M12, 12 months; PoNR, predictability of non-response; PoR, predictability of response. 


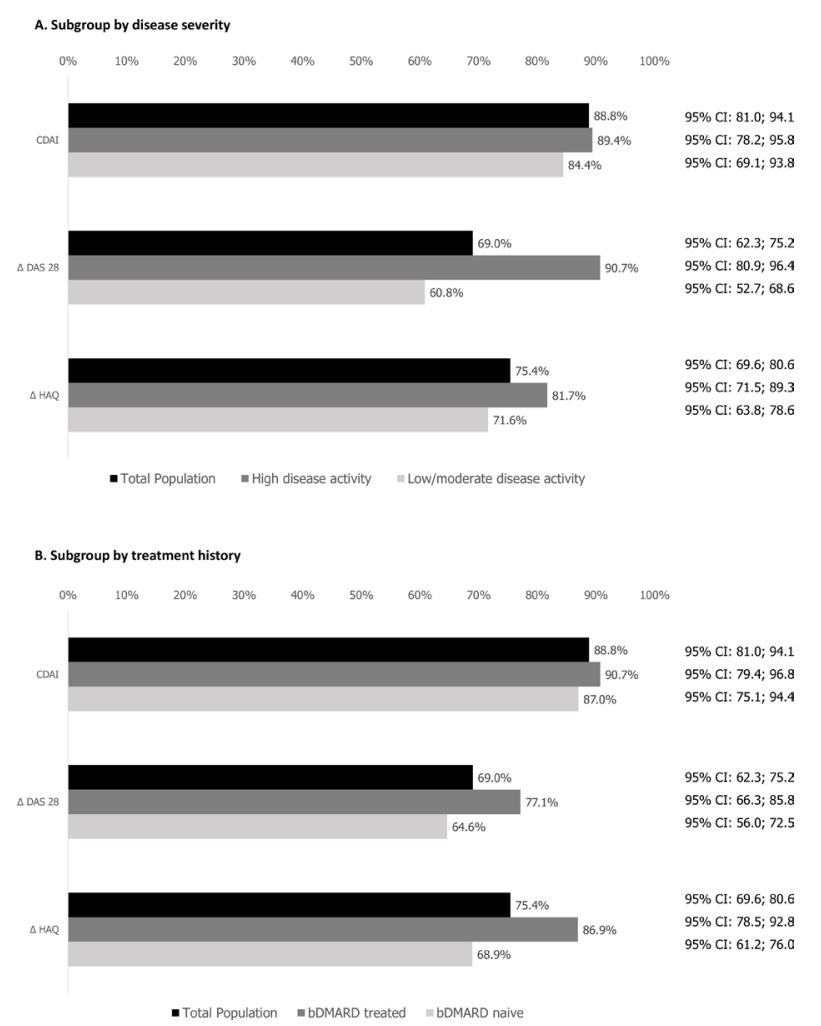

Figure 2 Non-response predictive values in subgroups of patients. CDAI, Clinical Disease Activity Index; CZP, certolizumab pegol; $\triangle \mathrm{DAS} 28$, change in 28-joint disease activity score; bDMARD, biological disease-modifying antirheumatic drug; $\triangle \mathrm{HAQ}-\mathrm{DI}$, change in Health Assessment Questionnaire Disability Index. High disease activity: DAS28(ESR) score $>5.1$ at baseline; low/moderate disease activity: DAS28(ESR) $\leq 3.2$ and $\leq 5.1$

specificity $(96 \%)$, the latter indicating that fewer than $5 \%$ of patients who achieved a CDAI response at M12 were non-responders at M3. This finding was consistent irrespective of disease severity at baseline and/or treatment history.

The real-world data reported here corroborate published clinical trial data. In the clinical study reported by Aletaha $e t a l,{ }^{14}$ for example, a principal observation was that failure to achieve minor clinical responses at M3 was a potential indicator of treatment failure at M6. Similarly, a good response at M3 was seen to be a reliable predictor of achieving the treatment goal at M6. Other clinical studies have also demonstrated the predictive value of early measures of disease activity and response to treatment, including clinical trials of CZP in patients with RA. ${ }^{11}{ }^{12}$ For example, in a post hoc analysis of the phase III RAPID-1 trial, ${ }^{13}$ which used identical definitions for early non-response and late treatment failure, the PoNR for the CDAI was $97 \% .^{11}$ The cohort of patients in ECLAIR that was most similar to those in RAPID-1 had high disease severity at baseline and had not been treated with bDMARDs previously; the PoNR for CDAI in this group was $87.5 \%$ (online supplementary table 2). Compared with the clinical trial setting, and the predictability of CDAI, DAS28(ESR) was less effective at predicting treatment failure at M12 in the overall ECLAIR population (although it was effective in the subgroup of patients with severe disease). This may be attributable to differences in the cut-offs used by each index to categorise disease activity ${ }^{25}$; however, there were also differences in baseline disease severity between the ECLAIR and RAPID-1 cohorts. While the ECLAIR population exhibited heterogeneous disease severity at baseline, with approximately $40 \%$ of patients having severe disease based on DAS28(ESR) score, the vast majority ( 99\%) of RAPID-1 patients fell within this category. Thus, the predictive utility of DAS28(ESR) in patients with severe disease was faithfully replicated in ECLAIR, although it appears that this index may not have sufficient sensitivity to predict treatment failure in those with moderate or low disease activity. Furthermore, it appears that CDAI may be more appropriate for predicting treatment failure in patients regardless of disease severity, pointing towards the utility of this index in a real-world setting.

A limitation of the study lies in the use of CDAI $>22$ to define non-response at 3 months. This may have excluded patients who experienced a reduction-even a large reduction-in CDAI while remaining above the threshold. Another possible limitation was the exclusion of about one-fifth of patients before the M3 visit, due to either permanent or temporary ( $\geq 2$ continuous weeks') discontinuation of CZP treatment. While missing data were imputed at M12, this was not feasible at M3 since this was the first timepoint following CZP initiation. It is not clear what impact the exclusion of these patients may have had. Furthermore, the choice of instruments (and cut-offs) for measuring disease activity, and the interpretation of these results in routine practice, remains difficult. Clearly, all thresholds used to measure treatment response are subject to debate, particularly for patients with longstanding disease. Nevertheless, the proportion of patients considered to have late treatment response remained between $30 \%$ and $50 \%$ in the present study, irrespective of the chosen criteria. Finally, it should be noted that while the evolution of treatment response over time (beyond M12) and long-term safety outcomes are clinically important considerations in longstanding diseases such as RA, these were not considered within the scope of this analysis.

In summary, we provide real-world evidence in support of clinical trial data, by demonstrating that high CDAI scores at M3 are a predictor of failure to achieve LDA at M12 in patients with RA initiating treatment with CZP. In addition, we observed high level of predictability with CDAI regardless of disease severity at baseline or treatment history, although this observation should be considered with caution in light of the low patient numbers in these subgroups. DAS28(ESR) also demonstrated good predictability, but only in patients with high disease activity at baseline and/or those previously treated with a bDMARD. As a simple tool for physicians to use in daily practice, we recommend the use of CDAI to evaluate treatment response after 3 months as a means to identify 
patients who are unlikely to achieve LDA and for whom an alternative therapy might be considered.

\section{Author affiliations}

${ }^{1}$ Rheumatology, CHU de la Cavale-Blanche, Brest, France

${ }^{2}$ UMR Inserm 1227, LabEx IGO, Université de Brest, Brest, France

${ }^{3}$ Rheumatology, Université de Lille, Lille, France

${ }^{4}$ CEMKA-EVAL, Bourg-La-Reine, France

${ }^{5}$ Institut Phisquare, Paris, France

${ }^{6}$ Centre d'Investigation Clinique-INSERM CIC 1431, CHU de Besançon, Besançon, France

${ }^{7}$ UCB Pharma, Colombes, France

${ }^{8} \mathrm{Mapi}$, an ICON company, Lyon, France

${ }^{9}$ Rheumatology, CHU Montpellier, Université de Montpellier, Montpellier, France

\section{Twitter Alain Saraux @alain.saraux}

Acknowledgements The authors thank the patients and their caregivers in addition to the investigators and their teams who contributed to this study. The authors also acknowledge Susanne Wiegratz, UCB Pharma GmbH, Monheim, Germany for publication coordination, and Vanessa Omnou, MSc, Costello Medical Consulting, London, UK, for editorial assistance. This study was funded by UCB Pharma.

Contributors Substantial contributions to study conception and design: AS, R-MF, FF, JM, GC, J-MJ, PH-M, BC. Substantial contributions to analysis and interpretation of the data: AS, R-MF, FF, JM, GC, J-MJ, PH-M, BC. Drafting the article or revising it critically for important intellectual content: AS, R-MF, FF, JM, GC, J-MJ, PH-M, BC. Final approval of the version of the article to be published: AS, R-MF, FF, JM, GC, J-MJ, PH-M, BC.

Funding This study was funded by UCB Pharma.

Competing interests $\mathrm{BC}$, AS and R-MF received honoraria from AbbVie, BMS, Lilly, Merck, Pfizer, Roche-Chugai, Sanofi, UCB Pharma. AS, R-MF, FF, JM, PH-M and $\mathrm{BC}$ were members of the scientific committee. GC and J-MJ are employed by UCB Pharma.

Patient consent for publication Obtained.

Provenance and peer review Not commissioned; externally peer reviewed. Data availability statement Data are available on reasonable request.

Open access This is an open access article distributed in accordance with the Creative Commons Attribution Non Commercial (CC BY-NC 4.0) license, which permits others to distribute, remix, adapt, build upon this work non-commercially, and license their derivative works on different terms, provided the original work is properly cited, appropriate credit is given, any changes made indicated, and the use is non-commercial. See: http://creativecommons.org/licenses/by-nc/4.0/.

ORCID iDs

Alain Saraux http://orcid.org/0000-0002-8454-7067

Bernard Combe http://orcid.org/0000-0003-4002-1861

\section{REFERENCES}

1 Singh JA, Saag KG, Bridges SL, et al. 2015 American College of Rheumatology guideline for the treatment of rheumatoid arthritis Arthritis Care Res 2016;68:1-25.

2 Daien C, Hua C, Gaujoux-Viala C, et al. Update of French Society for Rheumatology recommendations for managing rheumatoid arthritis. Joint Bone Spine 2019;86:135-50.

3 Smolen JS, Landewé R, Bijlsma J, et al. EULAR recommendations for the management of rheumatoid arthritis with synthetic and biological disease-modifying antirheumatic drugs: 2016 update. Ann Rheum Dis 2017:76:960-77.

4 Smolen JS, Aletaha D, Koeller M, et al. New therapies for treatment of rheumatoid arthritis. The Lancet 2007;370:1861-74.

5 Aga A-B, Lie E, Uhlig T, et al. Time trends in disease activity, response and remission rates in rheumatoid arthritis during the past decade: results from the NOR-DMARD study 2000-2010. Ann Rheum Dis 2015;74:381-8.

6 Orme ME, Macgilchrist KS, Mitchell S, et al. Systematic review and network meta-analysis of combination and monotherapy treatments in disease-modifying antirheumatic drug-experienced patients with rheumatoid arthritis: analysis of American College of Rheumatology criteria scores 20, 50, and 70. Biologics 2012;6:429-64.

7 Hetland ML, Christensen IJ, Tarp U, et al. Direct comparison of treatment responses, remission rates, and drug adherence in patients with rheumatoid arthritis treated with adalimumab, etanercept, or infliximab: results from eight years of surveillance of clinical practice in the nationwide Danish DANBIO registry. Arthritis Rheum 2010;62:22-32.

8 Smolen JS, Breedveld FC, Burmester GR, et al. Treating rheumatoid arthritis to target: 2014 update of the recommendations of an international task force. Ann Rheum Dis 2016;75:3-15.

9 Radner $\mathrm{H}$, Aletaha D. Anti-TNF in rheumatoid arthritis: an overview. Wien Med Wochenschr 2015;165:3-9.

10 Wijbrandts CA, Tak PP. Prediction of response to targeted treatment in rheumatoid arthritis. Mayo Clinic Proceedings 2017;92:1129-43.

11 Berenbaum F, Pham T, Claudepierre P, et al. Early non-response to certolizumab pegol in rheumatoid arthritis predicts treatment failure at one year. Data from a randomised phase III clinical trial. Joint Bone Spine 2018;85:59-64.

12 van der Heijde D, Keystone EC, Curtis JR, et al. Timing and magnitude of initial change in disease activity score 28 predicts the likelihood of achieving low disease activity at 1 year in rheumatoid arthritis patients treated with certolizumab pegol: a post-hoc analysis of the RAPID 1 trial. J Rheumatol 2012;39:1326-33.

13 Keystone E, Heijde DVD, Mason D, et al. Certolizumab pegol plus methotrexate is significantly more effective than placebo plus methotrexate in active rheumatoid arthritis: findings of a fifty-twoweek, phase III, multicenter, randomized, double-blind, placebocontrolled, parallel-group study. Arthritis Rheum 2008;58:3319-29.

14 Aletaha D, Alasti F, Smolen JS. Optimisation of a treat-to-target approach in rheumatoid arthritis: strategies for the 3-month time point. Ann Rheum Dis 2016;75:1479-85.

15 Keystone EC, Breedveld FC, Kupper H, et al. Long-term use of adalimumab as monotherapy after attainment of low disease activity with adalimumab plus methotrexate in patients with rheumatoid arthritis. RMD Open 2018;4:e000637.

16 Stoffer MA, Schoels MM, Smolen JS, et al. Evidence for treating rheumatoid arthritis to target: results of a systematic literature search update. Ann Rheum Dis 2016;75:16-22.

17 Aletaha D, Smolen J. The simplified disease activity index (SDAI) and the clinical disease activity index (CDAl): a review of their usefulness and validity in rheumatoid arthritis. Clin Exp Rheumatol 2005:23:S100-8.

18 Aletaha D, Nell VPK, Stamm T, et al. Acute phase reactants add little to composite disease activity indices for rheumatoid arthritis: validation of a clinical activity score. Arthritis Res Ther 2005; 7:R796-806.

19 Prevoo MLL, Van'T Hof MA, Kuper HH, et al. Modified disease activity scores that include twenty-eight-joint counts. Development and validation in a prospective longitudinal study of patients with rheumatoid arthritis. Arthritis Rheum 1995;38:44-8.

20 Fries JF, Spitz P, Kraines RG, et al. Measurement of patient outcome in arthritis. Arthritis Rheum 1980;23:137-45.

21 Curtis JR, Luijtens K, Kavanaugh A. Predicting future response to certolizumab pegol in rheumatoid arthritis patients: features at 12 weeks associated with low disease activity at 1 year. Arthritis Care Res 2012;64:658-67.

22 Curtis JR, Yang S, Chen L, et al. Predicting low disease activity and remission using early treatment response to antitumour necrosis factor therapy in patients with rheumatoid arthritis: exploratory analyses from the TEMPO trial. Ann Rheum Dis 2012;71:206-12.

23 Keystone EC, Curtis JR, Fleischmann ROYM, et al. Rapid improvement in the signs and symptoms of rheumatoid arthritis following certolizumab pegol treatment predicts better longterm outcomes: post-hoc analysis of a randomized controlled trial. $J$ Rheumatol 2011;38:990-6.

24 lannone F, Carlino G, Marchesoni A, et al. Early clinical response predicts low disease activity at one year in rheumatoid arthritis patients on treatment with certolizumab in real-life settings. An appraisal of the Italian registry GISEA. Joint Bone Spine 2016;83:721-5

25 Martins FM, da Silva JAP, Santos MJ, et al. DAS28, CDAl and SDA cut-offs do not translate the same information: results from the rheumatic diseases Portuguese register Reuma.pt. Rheumatology 2015;54:286-91. 